\title{
Evaluating causative agents, mortality factors, and laboratory data of hospital-acquired pneumonia patients
}

\author{
Emine Oznur ${ }^{1} \odot$, Seda Guzeldag² $\odot$, Nuri Cakir ${ }^{3 *}$ (1)
}

\begin{abstract}
SUMMARY
OBJECTIVE: In the recent years, the increase in death rates from nosocomial pneumonia draws attention. The aim of this study was to examine the causative agents and mortality factors of patients with pneumonia who were followed up in the chest diseases intensive care unit. METHODS: Data of 1070 patients with pneumonia were screened for this study. A total of 160 patients with hospital-acquired pneumonia included in this study. The relationship between factors such as patients' comorbidities, length of stay in the intensive care unit, history of hospitalization or respiratory support therapy, infection markers such as C-reactive protein, white blood cell, nutritional markers such as albumin and protein, renal and liver function tests, culture growing microorganisms, and clinical pulmonary infection scores was evaluated and mortality rates were examined.

RESULTS: Among 1070 patients, the rate of hospital-acquired pneumonia was $14.9 \%$, and the mortality rate of pneumonia was $16.9 \%$. Mortality was significantly increased in patients who stayed in the intensive care unit for more than 10 days, in patients with a clinical pulmonary infection score of $\geq 6$ and with a history of hospitalization in the past one month, and received invasive mechanical ventilation therapy. Mortality increased in patients with hypoalbuminemia, hypoproteinemia, and high C-reactive protein values. The most commonly grown microorganism was Acinetobacter baumannii, which was also found significantly in patients who underwent invasive mechanical ventilation.

CONCLUSION: In the clinical approach to hospital-acquired pneumonia, in order to prevent mortalities, it is important to reveal whether the newly emerging symptoms and signs are related to pneumonia, to identify the causative pathogen, and to determine the severity of the disease.

KEYWORDS: Hospital acquired pneumonia. Intensive care unit. Mortality.
\end{abstract}

\section{INTRODUCTION}

Hospital-acquired pneumonia (HAP) is the most common hospital-acquired infection ${ }^{1}$. HAP is defined as pneumonia that usually develops $48 \mathrm{~h}$ after hospitalization without incubation period at the time of hospitalization and that occurs within $48 \mathrm{~h}$ of discharge from the hospital ${ }^{2}$.

The time of onset of pneumonia emerges as an important variable in the prognosis of HAP patients in terms of both epidemiological and problematic microbiological factors. Early-onset HAP that develops within the first 4 days after hospitalization is generally benign and has a good prognosis due to treatable microorganisms. Late-onset HAP is the disease that develops on the fifth and subsequent days, with serious pathogens that show multidrug resistance (MDR), cause treatment problems, and increase treatment $\operatorname{cost}^{3-5}$. Microorganisms in the etiology of HAP may vary with the

${ }^{1}$ City Hospital, Department of Chest Diseases - Kayseri, Turkey.

${ }^{2}$ City Hospital, Department of Critical Care Medicine - Kayseri, Turkey.

${ }^{3}$ Bünyan State Hospital, Clinical Microbiology - Kayseri, Turkey.

*Corresponding author: nuricakir@gmail.com

Conflicts of interest: the authors declare there is no conflicts of interest. Funding: none.

Received on August 10, 2021. Accepted on September 20, 2021. 
underlying disease, presence of risk factors, and the time of occurrence of pneumonia ${ }^{6}$.

The aim of this study was to examine the causative agents, mortality factors, the contribution of laboratory data to clinical outcome and mortality of patients, and the contribution of clinical pulmonary infection score (CPIS) to the prognosis of the pneumonia patients who were followed up in the Chest Diseases Intensive Care Unit of Erciyes University Faculty of Medicine.

\section{METHODS}

This study was carried out retrospectively in "hospital-developing pneumonia" patients who stayed in the Chest Diseases Intensive Care Unit of Erciyes University Medical Faculty Hospital between June 2006 and June 2011 for more than 48 h, who were known to have no previous pneumonia, and whose secretions increased in the first $48 \mathrm{~h}$ of discharge. This study was approved by the Research Ethics Committee of Erciyes University Medical Sciences (resolution number: 2011-361).

The registry archive of the chest diseases intensive care unit (ICU) was scanned for the study. The profiles, file numbers, and clinical and laboratory data of the 1070 patients were obtained. Hospitalization history of these patients before their epicrisis, antibiotics usage before admission to intensive care, comorbidities, course of fever, length of stay in the ICU, CPIS value, mortality, facilitating risk factors, clinical navigational information, etiological microorganisms, empirical antibiotics usage, and antibiotic susceptibilities were recorded. To support the data, the control records of the infection committee were scanned with the permission of the infectious diseases control committee. Patients who were discharged from the ICU in the first $24 \mathrm{~h}$ were excluded from the study. Liver and renal function tests, albumin, protein, magnesium $(\mathrm{Mg})$, C-reactive protein (CRP) levels, and complete blood count parameters were recorded. The risk factors for HAP such as the presence of central venous catheter, tracheostomy, reintubation, bronchoscopy, tube thoracoscopy, percutaneous endoscopic gastrostomy (PEG), urethral catheter, nasogastric tube, history of total parenteral nutrition (TPN), cytostatic agents, transfusion, hypertension, diabetes mellitus, chronic obstructive pulmonary disease, underlying malignancy, central nervous system pathology, bronchiectasis, hemodialysis admission, metabolic acidosis, and length of stay in ICU were recorded and factors affecting mortality rates were examined.

\section{Statistical analysis}

Data analysis was done using Statistical Package for Social Science version 11.5 package program. Shapiro-Wilk test was used for the normally distributed continuous data. Descriptive statistics were presented as number of cases, median, and lowest and highest values for continuous variables and (\%) for categorical variables. Categorical variables were evaluated with Pearson's chi-square or Fisher's exact test. Continuous variables were analyzed by univariate logistic regression analysis. As a result of univariate statistical analyses, variables determined as $\mathrm{p}<0.25$ were accepted as candidate risk factors in distinguishing survivors and exitus groups. Among all possible risk factors, the most determining factors were investigated by multivariate logistic regression analysis. Odds ratio and $95 \%$ confidence interval for each variable were calculated. For $\mathrm{p}<0.05$, all the results were considered statistically significant.

\section{RESULTS}

The names and protocol numbers of 1070 patients were obtained from the records of the Intensive Care Unit of Erciyes University Faculty of Medicine. Out of 1070, 226 patients were excluded from this study because they were discharged from the ICU or died in the first $24 \mathrm{~h}$. Of the 844 patients whose records could be accessed, 160 (18.9\%) patients were identified with microorganisms growing $48 \mathrm{~h}$ after they were admitted to the ICU and/ or in the first $48 \mathrm{~h}$ of discharge. The clinical and demographic data of these 160 patients who met all the study criteria were examined. CRP, white blood cell (WBC), blood urine nitrogen (BUN), creatinine, aspartate transaminase (AST), analine transaminase (ALT), and Mg values at the date of reproduction of the microorganisms were recorded from the database.

Among 160 patients, 97 (60.6\%) were males and 63 (39.4\%) were females. The mortality rate was $61.9 \%(\mathrm{n}=99)$. Of 99 patients, $60(60.6 \%)$ were males and 39 (39.4\%) were females ( $\mathrm{p}=0.959)$. A total of 115 (71.9\%) patients who developed HAP were $\geq 65$ years of age. Over $75.8 \%$ ( $n=75)$ of the patients who died during the hospitalization were $\geq 65$ years. The difference between the rates of mortality in $\geq 65$ years was not statistically different $(\mathrm{p}=0.353)$.

Considering underlying diseases, $53.1 \%(\mathrm{n}=85)$ of the patients had chronic obstructive pulmonary disease, $22.5 \%$ ( $\mathrm{n}=36)$ had hypertension, 16.9\% $(\mathrm{n}=27)$ had diabetes mellitus, and $4.4 \%(\mathrm{n}=7)$ had bronchiectasis. Central nervous system pathology was present in $11(6.9 \%)$ patients. Mortality rates were examined and we found that $27.7 \%(\mathrm{n}=26)$ patients had hypertension, $53.7 \%(\mathrm{n}=51)$ had chronic obstructive pulmonary disease, $9.7 \%(\mathrm{n}=9)$ had underlying malignancy, $17.9 \%$ $(\mathrm{n}=17)$ had hemodialysis admission, $17.7 \%(\mathrm{n}=17)$ had diabetes mellitus, $7.3 \%(\mathrm{n}=7)$ had central nervous system pathology, and $1.0 \%(\mathrm{n}=1)$ had bronchiectasis. Bronchiectasis and hemodialysis admission were significantly associated with mortality $(\mathrm{p}=0.013$ and $\mathrm{p}=0.022$, respectively) (Table 1 ).

Notably, $131(81.9 \%)$ patients were treated in invasive mechanical ventilation (IMV) and $62(38.8 \%)$ patients were 
treated in non-invasive mechanical ventilation (NIMV). The number of patients receiving only oxygen therapy was 7 (4.4\%). In addition, $90.9 \%$ of the deceased patients were treated in IMV $(\mathrm{n}=90)$, and three patients received oxygen therapy during their hospitalization. There was a statistically significant relationship between IMV and mortality $(\mathrm{p}<0.001)$ (Table 1$)$.

Effects of laboratory parameters on mortality rates are given in Table 2. The difference between WBC levels in two groups was not statistically significant $(\mathrm{p}=0.254)$. However, the difference between mean CRP levels of two groups and its relation with mortality was statistically significant $(\mathrm{p}=0.003)$. Although the mean protein values were $5.2(2.3-7.7)$, there was a weak statistically significant correlation with mortality $(\mathrm{p}=0.049)$. The mean albumin value of the patients in exitus group was 2.3 (1.0-3.8), and its relationship with mortality was also considered statistically significant $(\mathrm{p}=0.02)$. No significant correlations were found between other laboratory values and mortality.
Among 86 patients whose CPIS could be calculated, the mean CPIS value of the surviving patients was 7 (3-10), while the mean CPIS value of the deceased patients was eight (4-10). The correlation between CPIS level and mortality was statistically significant $(\mathrm{p}=0.013)$. Of 86 patients whose CPIS value was calculated, 59 died and 57 had a CPIS value of $\geq 6$ (96.6\%). This result showed that there was a statistically significant relationship between mortality and CPIS $\geq 6$ ( $p=0.004)$.

While a single microorganism was found to be responsible in 131 cultures, it was recorded as polymicrobial in 29 cultures. Samples were taken from endotracheal aspirate (ETA) $(\mathrm{n}=108)$, bronchoalveolar lavage (BAL) fluid $(\mathrm{n}=31)$, and sputum $(\mathrm{n}=21)$. Pneumonia agent was also isolated in blood culture in 16 out of 160 patients who developed HAP. These isolates were Acinetobacter baumannii ( $\mathrm{n}=9)$, Staphylococcus aureus $(\mathrm{n}=3)$, Escherichia coli $(\mathrm{n}=2)$, Klebsiella pneumoniae $(\mathrm{n}=1)$, and Enterococcus faecium $(\mathrm{n}=1)$. The most commonly isolated microorganisms were, in order of frequency, A. baumannii $(\mathrm{n}=71)$,

Table 1. Effects of underlying diseases and respiratory support on mortality rates.

\begin{tabular}{|c|c|c|c|c|}
\hline & Alive & Exitus & $p$-value & OR $(95 \% \mathrm{Cl})$ \\
\hline \multicolumn{5}{|c|}{ Hypertension, n (\%) } \\
\hline$(+)$ & $48(82.8)$ & $68(72.3)$ & \multirow{2}{*}{0.142} & \multirow{2}{*}{$1.835(0.810-4.157)$} \\
\hline$(-)$ & $10(17.2)$ & $26(27.7)$ & & \\
\hline \multicolumn{5}{|c|}{ Chronic obstructive pulmonary disease, $\mathrm{n}(\%)$} \\
\hline$(+)$ & $25(42.4)$ & $44(46.3)$ & \multirow{2}{*}{0.632} & \multirow{2}{*}{$0.852(0.443-1.641)$} \\
\hline$(-)$ & $34(57.6)$ & $51(53.7)$ & & \\
\hline \multicolumn{5}{|c|}{ Malignancy, n (\%) } \\
\hline$(+)$ & $55(94.8)$ & $84(90.3)$ & \multirow{2}{*}{0.373} & \multirow{2}{*}{$1.964(0.509-7.578)$} \\
\hline$(-)$ & $3(5.2)$ & $9(9.7)$ & & \\
\hline \multicolumn{5}{|c|}{ Diabetes mellitus, n (\%) } \\
\hline$(+)$ & $49(83.1)$ & $79(82.3)$ & \multirow{2}{*}{0.904} & \multirow{2}{*}{$1.054(0.447-2.488)$} \\
\hline$(-)$ & $10(16.9)$ & $17(17.7)$ & & \\
\hline
\end{tabular}

Central nervous system pathology, $\mathrm{n}(\%)$

\begin{tabular}{l|c|c|c|c}
\hline$(+)$ & $55(93.2)$ & $89(92.7)$ & \multirow{2}{*}{1.000} & \multirow{2}{*}{$1.081(0.303-3.865)$} \\
\cline { 1 - 2 }$(-)$ & $4(6.8)$ & $7(7.3)$ & \\
\cline { 1 - 2 } Bronchiectasis, n (\%) & $53(89.8)$ & $95(99.0)$ & \multirow{2}{*}{0.013} & $0.093(0.011-0.793)$ \\
\cline { 1 - 2 } & $6(+)$ & $1(1.0)$ & & \\
\hline
\end{tabular}

Hemodialysis admission, $\mathrm{n}(\%)$

\begin{tabular}{c|c|c|c|c}
\hline \multicolumn{1}{|c|}{$(+)$} & $56(94.9)$ & $78(82.1)$ & \multirow{2}{*}{0.022} & \multirow{2}{*}{$4.068(1.137-14.552)$} \\
\cline { 1 - 2 }$(-)$ & $3(5.1)$ & $17(17.9)$ & & \\
\cline { 1 - 2 } Invasive mechanical ventilation, n (\%) & $40(67.8)$ & $90(90.9)$ & $<0.001$ & $4.750(1.978-11.408)$ \\
\hline Oxygen therapy, n (\%) & $4(6.8)$ & $3(3.0)$ & 0.426 & $0.430(0.093-1.991)$ \\
\hline
\end{tabular}

OR: odds ratio; $\mathrm{Cl}$ : confidence interval. 
E. coli $(\mathrm{n}=28)$ (extended spectrum beta-lactamase [ESBL] production; $\mathrm{n}=18)$, Pseudomonas spp. ( $\mathrm{n}=25)$, Klebsiella spp. $(\mathrm{n}=17)$, S. aureus $(\mathrm{n}=17)$ (methicillin-susceptible [MSSA] $\mathrm{n}=6$ and methicillin resistant $[\mathrm{MRSA}] \mathrm{n}=11)$, Gram (-) bacilli $(\mathrm{n}=12)$, Proteus spp. ( $\mathrm{n}=6)$, Streptococcus spp. $(\mathrm{n}=4)$, Enterobacteriaceae spp. ( $\mathrm{n}=3$ ), and others (Stenotrophomonas maltophilia, Serratia marcescens, Citrobacter koseri, and fungal agents) $(\mathrm{n}=15)$. Proteus spp. was found as a candidate risk factor for mortality $(\mathrm{p}=0.085)$. However, there were no significant relationships between the other microorganisms grown in the cultures of the patients and their mortality rates (Table 3 ).

\section{DISCUSSION}

The defense mechanisms of the patients hospitalized in the ICU are generally impaired due to existing or accompanying diseases. Immune paralysis occurs due to the release of anti-inflammatory mediators and is associated with an increased risk of infectious complications. Immunosuppressive therapies, which are frequently applied to intensive care patients, contribute to these complications and convey patients to the high-risk group for nosocomial infection ${ }^{7}$. Endotracheal intubation reduces local defense mechanisms and paves the way for respiratory tract infections. In the first $24 \mathrm{~h}$ after admission to the ICU, the mouth is colonized by pathogenic bacteria ${ }^{8}$. In the first $48-72 \mathrm{~h}, 50 \%$ of the patients, and at the end of the first week almost all patients, are colonized by the factors that make up the flora of the unit. Colonization rate in critically ill patients reaches up to higher rates ${ }^{9}$. In nosocomial pneumonia, there is usually microaspiration or macroaspiration of these colonized microorganisms.

The main factors in early-onset HAP are not different from community-acquired pneumonia, and the most common are Streptococcus pneumoniae, Haemophilus influenzae, and MSSA ${ }^{1}$. In late-onset pneumonia, P. aeruginosa, Acinetobacter spp.,

Table 2. Effects of laboratory parameters and CPIS on mortality rates.

\begin{tabular}{l|c|c|c} 
& Alive Mean $(95 \% \mathrm{Cl})$ & Exitus Mean $(95 \% \mathrm{Cl})$ & p-value \\
\hline Albumin $(\mathrm{g} / \mathrm{dL})$ & $2.5(1.2-3.8)$ & $2.3(1.0-3.8)$ & 0.020 \\
\hline Total Protein $(\mathrm{g} / \mathrm{dL})$ & $5.3(3.9-7.6)$ & $5.2(2.3-7.7)$ & 0.049 \\
\hline $\mathrm{Mg}(\mathrm{mg} / \mathrm{dL})$ & $0.8(0.6-1.4)$ & $0.9(0.3-1.7)$ & 0.611 \\
\hline WBC $\left(\times 10^{3} / \mathrm{mm}^{3}\right)$ & $14(5-36)$ & $15(2-58)$ & 0.254 \\
\hline AST $(\mathrm{IU} / \mathrm{L})$ & $32(10-561)$ & $35(5-2040)$ & 0.370 \\
\hline ALT $(\mathrm{IU} / \mathrm{L})$ & $31.5(5-937)$ & $26(0.8-1614)$ & 0.863 \\
\hline BUN $(\mathrm{mg} / \mathrm{dL})$ & $30(9-86)$ & $32(9-131)$ & 0.096 \\
\hline Creatinine $(\mathrm{mg} / \mathrm{dL})$ & $1(0.4-2.8)$ & $1.1(0.3-38)$ & 0.144 \\
\hline CRP $(\mathrm{mg} / \mathrm{L})$ & $62(1.3-339)$ & $119(3-638)$ & 0.003 \\
\hline CPIS & $7(3-10)$ & $8(4-10)$ & 0.013 \\
\hline
\end{tabular}

WBC: White blood cell; AST: aspartate aminotransferase; ALT: alanine aminotransferase; BUN: blood urine nitrogen; CRP: C-reactive protein; Cl: confidence interval; CPIS: clinical pulmonary infection scores.

Table 3. Effects of microorganisms on patients' mortality rates.

\begin{tabular}{l|c|c|c} 
& Alive $(\mathrm{n}=59)$ & Exitus $(\mathrm{n}=99)$ & p-value \\
\hline Acinetobacter spp. (\%) & $23(39.0)$ & $46(46.5)$ & 0.359 \\
\hline E. coli (\%) & $9(15.3)$ & $19(19.2)$ & 0.531 \\
\hline Enterobacteriaceae spp. (\%) & $1(1.7)$ & $2(2.0)$ & 1.000 \\
\hline Gram (-) bacilli (\%) & $5(8.5)$ & $7(7.1)$ & 0.763 \\
\hline Klebsiella spp. (\%) & $6(10.2)$ & $11(11.1)$ & 0.853 \\
\hline Proteus spp. (\%) & - & $6(6.1)$ & 0.085 \\
\hline Pseudomonas spp. (\%) & $10(16.9)$ & $15(15.2)$ & 0.765 \\
\hline Staphylococcus aureus (\%) & $6(10.2)$ & $10(10.1)$ & 0.989 \\
\hline Streptococcus spp. (\%) & $2(3.4)$ & $2(2.0)$ & 0.630 \\
\hline Others (\%) & $8(13.6)$ & $7(7.1)$ & 0.178 \\
\hline
\end{tabular}


Enterobacter spp., Klebsiella spp., Gram-negative agents, and S. aureus can be seen as a factor in $20-30 \%$ of cases ${ }^{1,10}$. The prevalence of Legionella species is reported to be $10-20 \%$ and its frequency increases in cases of corticosteroid use, immunosuppression, and previous antibiotic use ${ }^{11}$. Legionella pneumophila should be considered among the causative agents of HAP in patients hospitalized in the ICU ${ }^{11}$.

The early or late stage of HAP, the underlying risk factors, and the severity of pneumonia shape the empirical treatment ${ }^{12}$. Appropriateness in empirical treatment is possible with the correct estimation of the origin organisms and requires rational antibiotic use skills that require clinical and microbiological knowledge, including local resistance data from the ICU.

Mortality rates of lower respiratory tract infections in hospitalized patients are high. Mortality is much higher in pneumonia developing especially in ventilator-dependent patients ${ }^{13}$. In our study considering underlying diseases, bronchiectasis and hemodialysis admission were significantly associated with mortality $(\mathrm{p}=0.013$ and $\mathrm{p}=0.022$, respectively) in HAP patients. There was also a statistically significant relationship between IMV and mortality $(\mathrm{p}<0.001)$.

It is believed that the most important cause of HAP is the colonization of the gastrointestinal tract and oropharynx by pathogenic microorganisms ${ }^{14,15}$. Pneumonia occurs with aspiration of these pathogens and failure of the host defense $\mathrm{e}^{14}$. Microaspiration of oropharyngeal secretions may occur during sleep in normal individuals. However, these aspirates are small and originated from nonpathogenic flora. However, in hospitalized patients, oropharyngeal colonization occurs with pathogenic aerobic Gram-negative bacilli. Colonization is facilitated by coma, hypotension, acidosis, azotemia, alcoholism, diabetes mellitus, leukocytosis, leukopenia, pulmonary disease, nasogastric and endotracheal intubation, and antibiotic utilization. In these patients, the aspirated volume and frequency of aspiration also increase for various reasons such as altered consciousness, difficulty in swallowing, decreased gurgling reflex, delayed gastric emptying, and slowing of gastrointestinal motility ${ }^{16}$. Normally, gastric acidity prevents pathogenic microorganisms. However, in cases of advanced age, achlorhydria, ileus, upper gastrointestinal system diseases, use of antacids or $\mathrm{H} 2$ receptor antagonists, proton-pump inhibitors, and enteral nutrition, the gastric $\mathrm{pH}$ rises above 4 and gastric acid protection disappears. In the HAP diagnosis and treatment management guidelines published by the American Thoracic Society in 2005, common pathogens in HAP cases are Gram-negative bacilli such as P. aeruginosa, E. coli, $K$. pneumoniae, and Acinetobacter spp. Gram-positive cocci, especially MRSA and anaerobes. In the 2008 HAP Guideline of the Turkish Thoracic Society, common pathogens in late-onset HAP were indicated as $P$. aeruginosa, Acinetobacter spp., Enterobacter spp., and Klebsiella spp. However, Hu et al. reported that agent spectrum and antibiotic susceptibility differ in late-onset Group 2 and Group 3 HAP cases ${ }^{17}$. The most common isolated microorganisms in our study were, in order of frequency, $A$. bauman$n i i(\mathrm{n}=71)$ and $E$. coli $(\mathrm{n}=28)$ (ESBL production; $\mathrm{n}=18)$. Proteus spp. $(\mathrm{n}=6)$ was found as a candidate risk factor for mortality $(p=0.085)$. However, there were no significant relationships between the other microorganisms grown in the cultures of the patients and their mortality rates (Table 3 ).

Laboratory tests have been performed to complete the diagnosis and to guide clinicians about the prognosis of HAP. Zheng et al. showed that the neutrophil/lymphocyte count ratio, procalcitonin, and CRP levels were markedly different between the noninfection and HAP groups ${ }^{18}$. In our study, the effects of laboratory parameters on mortality rates were also examined and the difference between mean CRP and albumin levels of groups and their relations with mortality were found statistically significant $(\mathrm{p}=0.003$ and $\mathrm{p}=0.02$, respectively). We also examined the relation between CPIS and mortality and our study showed that there was a statistically significant relationship between mortality and CPIS $\geq 6(p=0.004)$.

Our study had some limitations. First, this was a retrospective study evaluating causative agents, mortality factors, laboratory data to clinical outcome and mortality of patients, and the contribution of CPIS to prognosis of patients with HAP. Therefore, our study could be improved by performing further prospective studies. Second, we examined a limited number of laboratory parameters and inflammation markers. Further prospective studies are required to clarify the effects of laboratory parameters and inflammation markers on prognosis and mortality rates of patients with HAP.

\section{CONCLUSIONS}

The clinical diagnosis of HAP is difficult. Infectious and noninfectious pathologies should be considered in the differential diagnosis. Difficulty in diagnosis causes unnecessary antibiotic utilization, and as a result, there is an increase in risk of antibiotic-resistant bacterial infection, toxicity, and treatment cost. In addition, mortality rates of these patients are high. Hence, combining clinical, laboratory, and CPIS data and isolating the related pathogens earlier with appropriate antibiotics therapy may help clinicians to predict the prognosis of patients with HAP.

\section{AUTHORS' CONTRIBUTION}

EO: Conceptualization, Data curation, Formal analysis, Methodology, Writing - original draft. SG: Conceptualization, Data curation, Formal analysis, Resources, Software, Writing review \& editing. NC: Conceptualization, Formal analysis, Investigation, Methodology, Project administration, Software, Supervision, Validation, Visualization, Writing - review \& editing. 


\section{REFERENCES}

1. Kalil AC, Metersky ML, Klompas M, Muscedere J, Sweeney DA, Palmer LB, et al. Management of adults with hospitalacquired and ventilator-associated pneumonia: 2016 clinical practice guidelines by the infectious diseases society of America and the American thoracic society. Clin Infect Dis. 2016;63(5):e61-111. https://doi.org/10.1093/cid/ciw353

2. Puzniak L, Dillon R, Palmer T, Collings H, Enstone A. Systematic literature review of real-world evidence of ceftolozane/tazobactam for the treatment of respiratory infections. Infect Dis Ther. 2021;10(3):1227-52. https://doi.org/10.1007/s40121-021-00491-x

3. Bonine NG, Berger A, Altincatal A, Wang R, Bhagnani T, Gillard P, et al. Impact of delayed appropriate antibiotic therapy on patient outcomes by antibiotic resistance status from serious gramnegative bacterial infections. Am J Med Sci. 2019;357(2):10310. https://doi.org/10.1016/j.amjms.2018.11.009

4. Tabak YP, Merchant S, Ye G, Vankeepuram L, Gupta V, Kurtz SG, Puzniak LA. Incremental clinical and economic burden of suspected respiratory infections due to multi-drug-resistant Pseudomonas aeruginosa in the United States. J Hosp Infect. 2019;103(2):134-41. https://doi.org/10.1016/j.jhin.2019.06.005

5. Neidell MJ, Cohen B, Furuya Y, Hill J, Jeon CY, Glied S, Larson EL. Costs of healthcare- and community-associated infections with antimicrobial-resistant versus antimicrobial-susceptible organisms. Clin Infect Dis. 2012;55(6):807-15. https://doi. org/10.1093/cid/cis552

6. Kumar ST, Yassin A, Bhowmick T, Dixit D. Recommendations from the 2016 guidelines for the management of adults with hospital-acquired or ventilator-associated pneumonia. P T. 2017;42(12):767-72. PMID: 29234216

7. Chalmers JD, Taylor JK, Singanayagam A, Fleming GB, Akram AR, Mandal P, et al. Epidemiology, antibiotic therapy, and clinical outcomes in health care-associated pneumonia: a UK cohort study. Clin Infect Dis. 2011;53(2):107-13. https://doi.org/10.1093/cid/cir274

8. Safarabadi M, Ghaznavi-Rad E, Pakniyat A, Rezaie K, Jadidi A. Comparing the effect of echinacea and chlorhexidine mouthwash on the microbial flora of intubated patients admitted to the intensive care unit. Iran J Nurs Midwifery Res. 2017;22(6):481-5. https://doi.org/10.4103/ijnmr.IJNMR_92_16

9. Divatia JV, Pulinilkunnathil JG, Myatra SN. Nosocomial infections and ventilator-associated pneumonia in cancer patients. Oncologic Critical Care. 2019;1419-39. https://doi. org/10.1007/978-3-319-74588-6_125
10. Sharma L, Losier A, Tolbert T, Dela Cruz CS, Marion CR. Atypical pneumonia: updates on Legionella, Chlamydophila, and Mycoplasma pneumonia. Clin Chest Med. 2017;38(1):4558. https://doi.org/10.1016/j.ccm.2016.11.011

11. Brady MF, Sundareshan V. Legionnaires' Disease. $2021 \mathrm{Jul} 18$. In: StatPearls [Internet]. Treasure Island: StatPearls Publishing; 2021. PMID: 28613558

12. But A, Yetkin MA, Kanyilmaz D, Aslaner H, Baştuğ A, Aypak $A$, et al. Analysis of epidemiology and risk factors for mortality in ventilator-associated pneumonia attacks in intensive care unit patients. Turk J Med Sci. 2017;47(3):812-6. https://doi. org/10.3906/sag-1601-38

13. Canturan SY, Yilmazer N, Sarikaya R, Avsar Z, Ertek M, Uyaner I. Retrospective Assessment of Ventilator-Associated Pneumonias due to Acinetobacter baumannii in an Oncology Hospital. Sisli Etfal Hastan Tip Bul. 2021;55(2):193-6. https:// doi.org/10.14744/SEMB.2021.01700

14. Khaky B, Yazdannik A, Mahjobipoor H. Evaluating the efficacy of nanosil mouthwash on the preventing pulmonary infection in intensive care unit: a randomized clinical trial. Med Arch. 2018;72(3):206-9. https://doi.org/10.5455/ medarh.2018.72.206-209

15. Li L, Ai Z, Li L, Zheng X, Jie L. Can routine oral care with antiseptics prevent ventilator-associated pneumonia in patients receiving mechanical ventilation? An update meta-analysis from 17 randomized controlled trials. Int J Clin Exp Med. 2015;8(2):1645-57. PMID: 25932093

16. Pozuelo-Carrascosa DP, Herráiz-Adillo Á, Alvarez-Bueno C, Añón JM, Martínez-Vizcaíno V, Cavero-Redondo I. Subglottic secretion drainage for preventing ventilator-associated pneumonia: an overview of systematic reviews and an updated metaanalysis. Eur Respir Rev. 2020;29(155):190107. https://doi. org/10.1183/16000617.0107-2019

17. Hu BJ, Wei L, Zhang XZ, Tang YC, Ni YX, Li YM, et al. A retrospective cohort study of the influence of time of hospitalacquired pneumonia onset on pathogen constitution. Zhonghua Jie He He Hu Xi Za Zhi. 2005;28(2):112-6. PMID: 15854394

18. Zheng $N$, Zhu D, Han Y. Procalcitonin and C-reactive protein perform better than the neutrophil/lymphocyte count ratio in evaluating hospital acquired pneumonia. BMC Pulm Med. 2020;20(1):166. https://doi.org/10.1186/s12890020-01207-6 